\title{
Point-of-service diagnostic technology for detection of swine viral diseases
}

\author{
Lapo Nannucci ${ }^{1}$, Paolo Barattini ${ }^{2}$, Ioannis Bossis ${ }^{3}$, \\ Grzegorz Woźniakowski ${ }^{4}$, Gyula Balka ${ }^{5}$, Carolina Pugliese ${ }^{1}$ \\ ${ }^{1}$ Department of Agrifood Production and Environmental Sciences, \\ University of Florence 50144 Firenze, Italy \\ ${ }^{2}$ Kontor 46 di Matteo Bonasso SAS, 10125 Torino, Italy \\ ${ }^{3}$ Department of Animal Sciences, Agricultural University of Athens, 11855 Athens, Greece \\ ${ }^{4}$ Department of Swine Diseases, National Veterinary Research Institute, 24-100 Puławy, Poland \\ ${ }^{5}$ Department of Pathology, University of Veterinary Medicine, 1078 Budapest, Hungary \\ lapo.nannucci@unifi.it
}

Received: June 11, $2019 \quad$ Accepted: February 24, 2020

\begin{abstract}
Introduction: A research project is underway aiming to develop a field diagnostic tool for six important viruses of the pig sector, namely: African swine fever virus (ASFV), porcine reproductive and respiratory syndrome virus (PRRSV), swine influenza virus (SIV), porcine parvovirus (PPV), porcine circovirus (PCV2), and classical swine fever virus (CSFV). Material and Methods: To obtain a preliminary sounding of the interest in this type of instrument among its potential operators, a questionnaire was drawn up and submitted to three categories of stakeholders: farmers, veterinarians, and others (including scientific and technical staff working on animal farms). Four countries participated: Italy, Greece, Hungary, and Poland. Results: In total, 83 replies were collected and analysed in a breakdown by stakeholder type and pertinence, where the areas were the importance of the main diseases within the different countries, diagnostic tool operational issues, and economic issues. Conclusion: The main end-users of this kind of instrument are expected to be private veterinarians and pig producers. The infectious agents seeming to be most interesting to diagnose with the instrument are PRRSV, SIV, PPV, and PCV2. The most decisive parameters which have been selected by the stakeholders are sensitivity, cost, simplicity, and time required to obtain results. The economic issue analysis showed that the majority of those who would prefer to buy rather than rent the device are willing to pay up to $€ 3,000$ for a diagnostic field tool.
\end{abstract}

Keywords: pig farming, pig viral diseases, diagnostic tools, survey.

\section{Introduction}

The increased density in modern animal production systems has made them vulnerable to various transboundary infectious agents that threaten productivity, and the pork meat industry is just as much affected as others. Early diagnosis of and immediate establishment of reliable countermeasures to infectious diseases is essential to limit severe biophysical and socio-economic consequences.

Point of service (POS) diagnostic technologies for the detection of swine viral diseases directly in the field address these challenges and needs. A project is in progress aiming to develop a novel device based on advanced, proven bio-sensing and photonics technologies to tackle emerging and endemic viruses causing epidemics on swine farms in Europe that lead to sizeable financial damage. The diagnostic device will allow immediate threat assessment at the farm level, with the analytical quality of commercial and institutional laboratories. This device will be portable and provide results in $10 \mathrm{~min}$ for five samples simultaneously at a reasonable cost, making it highly suitable for use in the field. Its technology depends on antigen recognition by antibodies directed against selected swine viruses. The antibodies are immobilised on a nano-photonic structure, and the binding of the specific antigen to them leads to a change in the chemical and physical properties of the photonic resonating structure, expressed as a variation of their 
refractive index. The amount of shift in the light transmission response can be related to the concentration of the target antigen.

The research and development activities started on November $1^{\text {st }}, 2017$ and will be pursued for three and a half years, to allow enough time for the development and real-world validation of the technology.

The overall concept underpinning the project is that of a device for early field-based detection of important swine pathogens such as African swine fever virus (ASFV), porcine reproductive and respiratory syndrome virus (PRRSV), swine influenza virus (SIV), porcine parvovirus (PPV), porcine circovirus 2 (PCV2), and classical swine fever virus (CSFV).

The device will use swine oral fluid samples as its main input, although it will be compatible with other types of sample, such as faeces, blood serum, or nasal swabs. The use of oral fluid as the main input limits the time needed for the analysis and simplifies the sample collection, also allowing the collection of samples from free-living animals such as wild boars.

The initial project activities were aimed at identifying the main end-users or stakeholders and learning their requirements. The results of that research have been used as input to prepare the functional specifications of the diagnostic device and system. These requirements have been collected through a survey that has been written and refined by the Agricultural University of Athens, Greece, in collaboration with the National Veterinary Research Institute, Puławy, Poland, and subsequently distributed to stakeholders.

\section{Material and Methods}

During this initial phase of the project, potential end users were surveyed for their feedback. In collaboration with the other consortium partners and the stakeholders, this article's authors compiled an initial list of issues to be investigated by the questionnaire relevant to the scope of the Swinostics project. The issues were then translated into questions. The initial list of questions was too long and included some overlap and ambiguities, so that the time required to answer to the complete questionnaire was too long. Therefore, the final version had some parts cut, and it focused on the most relevant parameters that had been identified, with the aim of creating a questionnaire that required about $10 \mathrm{~min}$ to fully complete.

The final version consisted of 10 questions gathering information about swine diseases, diagnostic tool operational issues, and economic issues.

The identified users belong to different groups, mainly represented by pig producers, veterinarians, governmental organisations responsible for animal health and welfare, and researchers, hunters, and organisations associated with wildlife management.
The survey was shared with other partners of the consortium for further dissemination. The partners involved in the conducting of the survey among potential stakeholders (the Agricultural University of Athens (AUA), the National Veterinary Research Institute, Puławy (NVRI), the University of Florence (UNIFI), and the University of Veterinary Medicine of Budapest (UVMB)), will also carry out the future validation and clinical testing of the instrument in four different countries (Greece, Poland, Italy, and Hungary) not only on domestic pigs but also on semiwild fauna (open-range farms rearing the autochtonous Cinta Senese Tuscan pig breed) and wild fauna (boars).

The main end-user/stakeholder and the most representative group in the survey was that of pig producers and owners of pig commercial holdings. The commercial holdings were classified into two main groups: medium-size (100-500 sows) and large commercial holdings ( $>500$ sows). The interest of these end-users in the welfare of pigs and awareness of prevention and treatment of infectious diseases are forefront because of the direct economic impact of herd health on profitability and sustainability of their enterprises. The profitability of swine production is constantly undermined by the occurrence of endemic infectious diseases such as PRRS, SIV, PPV, and PCV2, and could be totally devastated by epidemic outbreaks of highly contagious and deadly diseases such as ASF. In such a scenario, the new diagnostic device may have a large impact on competence in early warning and containment of epidemics, monitoring of wild fauna reservoirs, and potentially on the profitability and management of pig production in Europe and worldwide.

The second main stakeholder group is that of veterinarians from the private sector. Their interest in having a convenient, reliable, and portable diagnostic technology for monitoring swine infectious diseases is also associated with profitable production of pigs and early recognition of pathologies. In terms of diagnosis, the possibility to perform this on-site and differentially is one of the most desirable elements, as important as sensitivity or the cost of a particular analysis. The differential diagnosis facilitated by the technology to be developed would aid animal healthcare providers in controlling important pig viral diseases. Due to the current situation with regards to ASF in Eastern Europe, especially in Poland and Hungary, the possibility to monitor this disease is exceptionally important. The same relates to SIV, CSF, PRRS, PCV2, or PPV, which are also targeted by the new diagnostic device.

The last main category of end-users was classified as others, including scientists and technicians in the field of veterinary diagnostics and animal health. Although this category of operator does not have as direct involvement in pig health management as veterinarians or pig farmers, it was nevertheless 
decided to include it in order to amass further useful information for the future setup phase of the instrument.

\section{Results}

The survey lasted for about two months: from early December 2017 until the end of January 2018. Hundreds of possible end-users and stakeholders were contacted, especially through the networks of the leaders of the pilot (parties carrying out field validation - PIWet, AUA, UNIFI, and UVMB). A total of 83 replies (79 fully completed questionnaires) were collected and analysed. The collected data were catalogued, and in the context of the amassed information, some questions were selected as more significant for the evaluation of the operators' interest in the development of the instrument.

Disaggregating the responses by stakeholder category and by country, it transpires that 15 pig farmers, 11 veterinarians, and 3 others participated from Italy; 4 pig farmers, 9 veterinarians, and 5 others gave input from Greece; 1 pig farmer, 10 veterinarians, and 2 others responded from Hungary; and 5 pig farmers, 15 veterinarians, and 2 others answered from Poland.

The selected information was further processed, so as to allow the most significant responses collected for each different country and different category of selected stakeholder groups to emerge. The questions were chosen on the basis of specific themes considered of the greatest interest, to sample the perceptions of the stakeholders regarding the use of field diagnostic tools. These themes were the importance of the main swine disease within the different countries, operational issues affecting diagnostic tools, and economic issues.

\section{Importance of the main diseases within the different countries}

The questionnaire included a monitor of how the stakeholders prioritised the diseases capable of causing economic losses in the pig breeding sector for their importance. The respondent was asked to rank the following pathologies in order of relevance: ASFV, CSFV, foot and mouth disease virus (FMDV), PRRSV, SIV, swine vesicular disease virus (SVDV), swine herpesvirus (Aujeszky's disease), swine hepatitis E virus (HepE), porcine epidemic diarrhoea virus (PEDV), transmissible gastroenteritis virus (TGEV), PCV2, and PPV.

The highest-priority disease appeared to be rather different in each of the four countries, reflecting the different spreading patterns of the diseases individually in different territories of the EU.

Italy. There is a clearly predominant stakeholder concern over PRRS. There are significant expressions of interest for a tool against PPV (13 pig producers out of 15 and 10 vets out of 11) (Fig.1), which is prevalent in the country. Another disease for which the development of a quick detection method would be welcome is PCV2 (selected by 12 pig producers out of 15 and 10 vets out of 11) (Fig. 1).

Furthermore, especially among pig farmers, there is a considerable interest in Aujeszky's disease diagnosis (11 pig producers out of 15) (Fig. 1), which is officially under the control of national animal health management entities (Local Health Authorities (ASL) and the Experimental Institute of Zooprophylaxis (IZS)).

Greece. In Greece, stakeholders appear to be interested in identifying SIV (seven vets out of nine and three pig producers out of four) (Fig. 1). PRRS diagnosis also seems to be very interesting to Greek stakeholders (six vets out of nine and three pig producers out of four) (Fig.1). Our questionnaire also highlighted an interest into Aujeszky's disease detection.

Hungary. PRRS also seems to be a very important disease for Hungarian stakeholders (eight vets out of nine) (Fig. 1). This correlates with an ongoing National Eradication Programme launched officially in 2014.

Hungarian vets also showed a notable level of interest in a tool for PCV2 (seven vets out of nine) (Fig. 1), demonstrating the significant domestic presence of the virus, which reaches $100 \%$ in commercial herds. In Hungary, also SIV seems to be an interesting disease to detect for the pig farming sector.

Poland. In Poland, due to the presence of ASF carried by herds of wild boars in some areas of the country, stakeholders showed high enthusiasm for a device dedicated to its pathology (15 vets out of 15 and 4 pig producers out of 5) (Fig. 1). Another important problem for Polish pig farms is PRRS (15 vets out of 15 and 5 pig producers out of 5) (Fig. 1), which is thus demonstrated to be the most important disease in all the four countries surveyed.

\section{Diagnostic tool operational issues}

The questionnaire also included an attempt to grasp the perceptions of stakeholders about the importance of the main technical and operational parameters of diagnostic tools. The questionnaire included the following items: sensitivity, specificity, repeatability/reproducibility, simplicity, time required for an analysis, cost, and portability. Stakeholders were asked to assign a score from 1 (extremely important) to 5 (not important) to each of these parameters, which are closely related to the functionality of the field diagnostic tools.

Analysing the obtained responses, it was noted that in all four countries, all stakeholder categories attributed high importance to sensitivity, even if in the 
case of Hungarian vets, this parameter appears secondary to the time necessary to obtain results (Fig. 2). Regarding cost, the importance of this parameter is evident especially among pig farmers. In fact, 11 out of 15 Italian farm operating respondents attributed high importance to cost as did 5 out of 5 in Poland (Fig. 2), while veterinarians considered this parameter less important, showing that the technicians working in animal health were willing to invest more money if necessary in order to obtain suitably performing instruments.

Another parameter that seems key for the stakeholders is the time needed to obtain the results, an aspect ranked as extremely important by 10 vets out of 11 and 9 breeders out of 15 in Italy and by 7 vets out of 9 in Hungary (Fig. 2.), demonstrating that in these countries animal health professionals are very interested in cutting the time necessary for analysis. In Poland and Greece, this parameter appeared to be considered less important.

Simplicity of use instead appears essential for Greek breeders, and their Italian counterparts agreed. They rated it as extremely important in 3 out of 5 cases in Greece and 12 out of 15 in Italy (Fig. 2).

\section{Economic issues}

The analysis of stakeholder predisposition to buy rather than rent a novel field diagnostic device shows that pig farmers are inclined to buy the instrument in all countries except Italy, where the number of those who would prefer rental is almost equal to the number of potential buyers (eight for purchase and seven for rental) (Fig. 3). In Italy, the majority of participating pig farmers were small or medium-sized enterprises, and during the survey's compilation, some of them showed interest in the possibility of buying a single instrument as a consortium of producers.

Regarding the other countries in general, the veterinarians would be more oriented towards renting the tool than the breeders, except in Poland where they showed a considerable tendency to choose a purchase (14 for purchase and 1 for rental) (Fig. 3).

Bearing on the sum the market would be willing to spend to buy a diagnostic tool, the questionnaire included an opportunity to choose which would be acceptable from the following prices: $€ 1,000-3,000$, up to $€ 5,000$, up to $€ 10,000$, or up to $€ 15,000$.

Analysing the responses, Italian breeders (five out of eight of whom were willing to spend from $€ 1,000$ to 3,000 ) seemed to be oriented to spending smaller amounts of money than veterinarians (two out of two of whom were willing to spend up to $€ 5,000)$ for this type of instrument. Regarding Hungary, there was only one participating pig farmer who was willing to spend up to $€ 5,000$, while the veterinarians seemed to be prepared for lower expenditure than Italian colleagues (two out of four Hungarian practitioners were willing to spend $€ 5,000$ and the other two from $€ 1,000$ to $€ 3,000)$. In Greece, stakeholders seemed to be inclined to spend more money than in the other countries, and in fact two pig farmers out of four and two veterinarians out of five were willing to pay up to $€ 10,000$ for this kind of technology. Regarding Poland, the majority of pig farmers (three out of five) could countenance spending up to $€ 5,000$ and one of them would pay up to $€ 10,000$, while the majority of veterinarians would like to invest smaller amounts (13 out of 15 were willing to spend from $€ 1,000$ to $€ 3,000$ ) in the diagnostic device (Fig. 4).
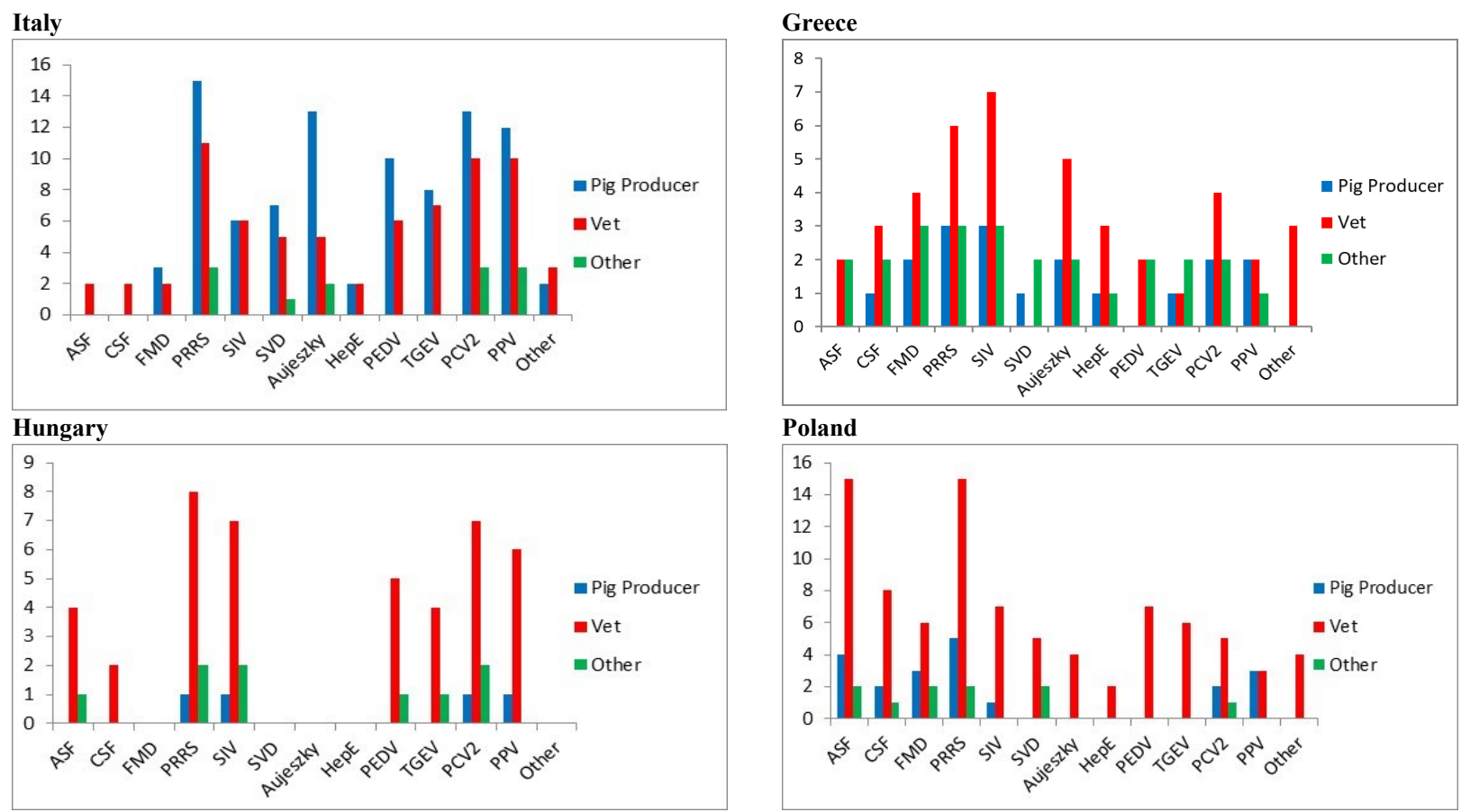

Fig. 1. Most important pathologies identified by the different categories of stakeholders 

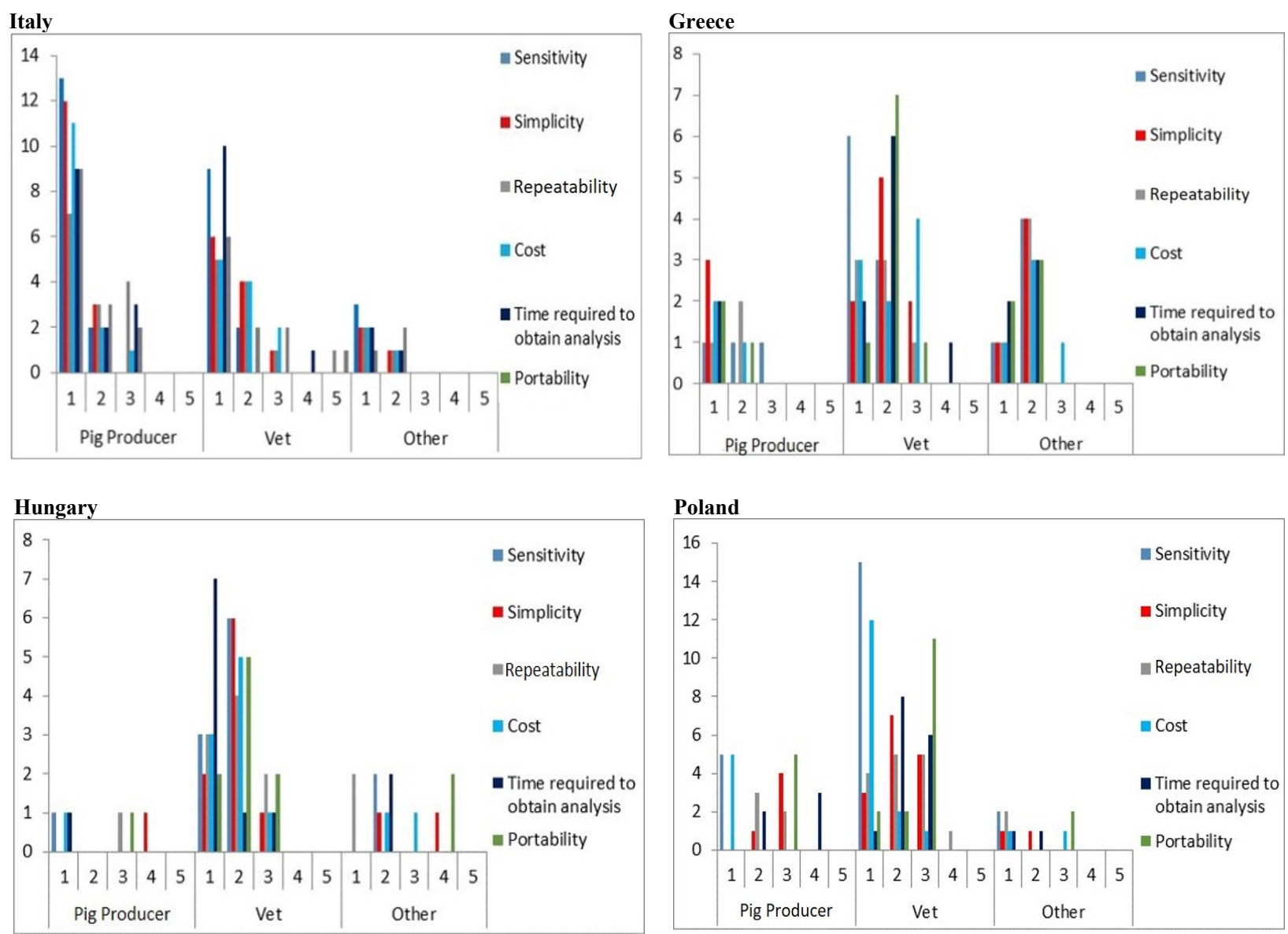

Fig. 2. Most important diagnostic tool operational parameters identified by the different categories of stakeholder
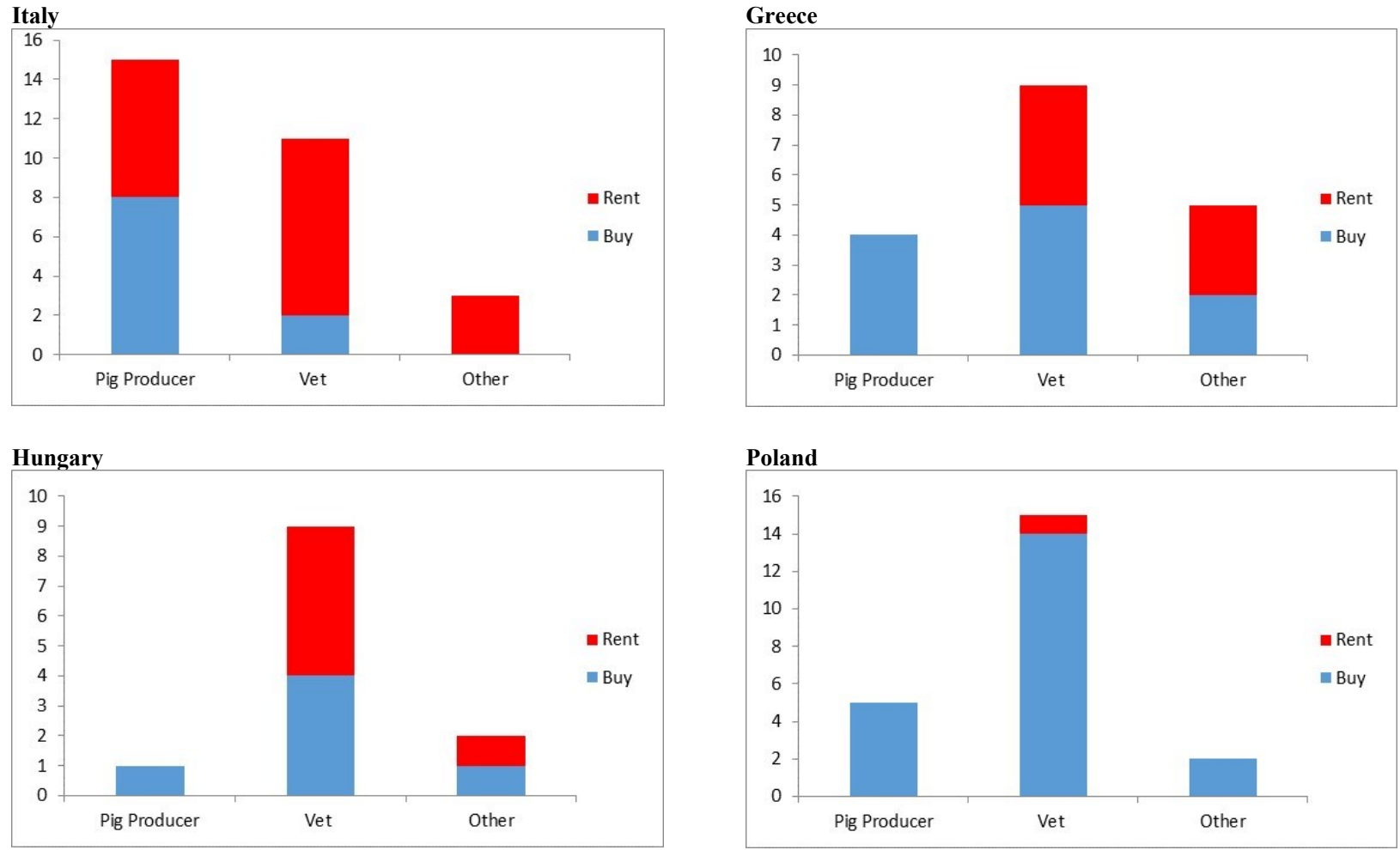

Fig. 3. Predispositions of the different categories of stakeholder from the four countries to buy or rent diagnostic field devices 
Regarding rental costs, the participating stakeholders had the choice of these different answers: $€ 20 /$ month, €50/month, €100/month, €150/month, or $€ 300 /$ month. The veterinarian stakeholder group appears to be accepting of more outlay for this type of service. In Italy, the acceptable spend was up to $€ 300$ per month in
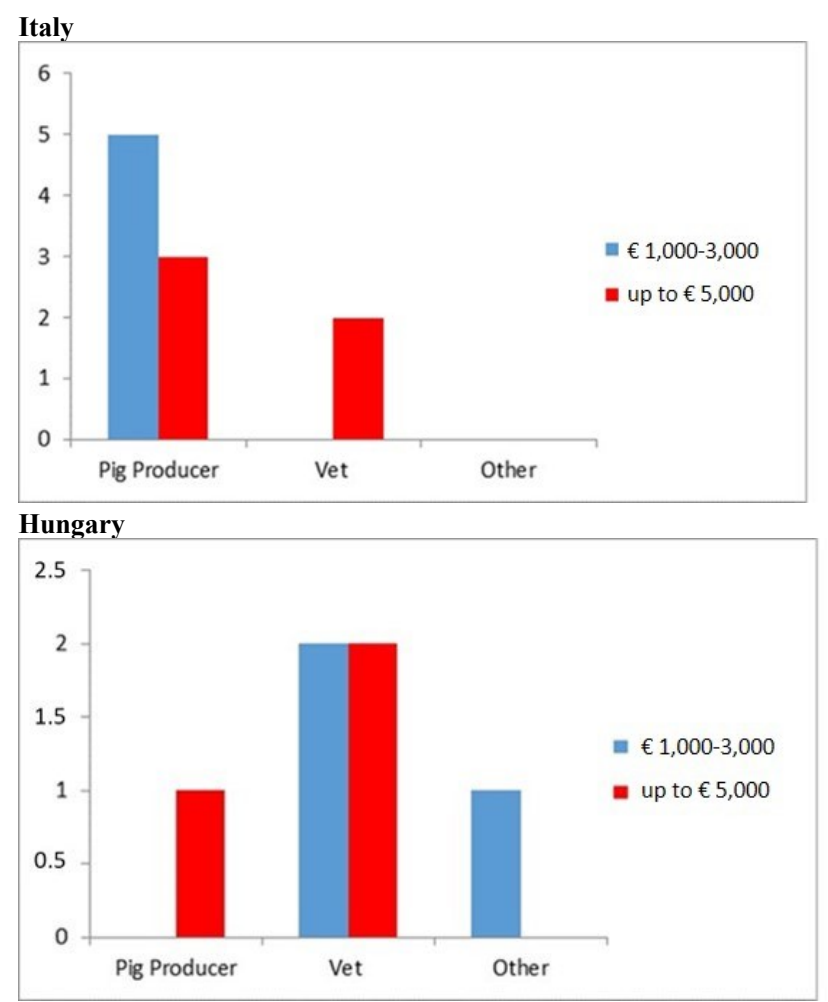

one case, up to $€ 100$ in two cases and up to $€ 20$ in five cases. Compared to other countries, in Italy, there was more widespread preparedness among breeders to rent the Swinostics device, but they were willing to spend less than animal health professionals (up to $€ 50$ in only two cases) (Fig. 5).
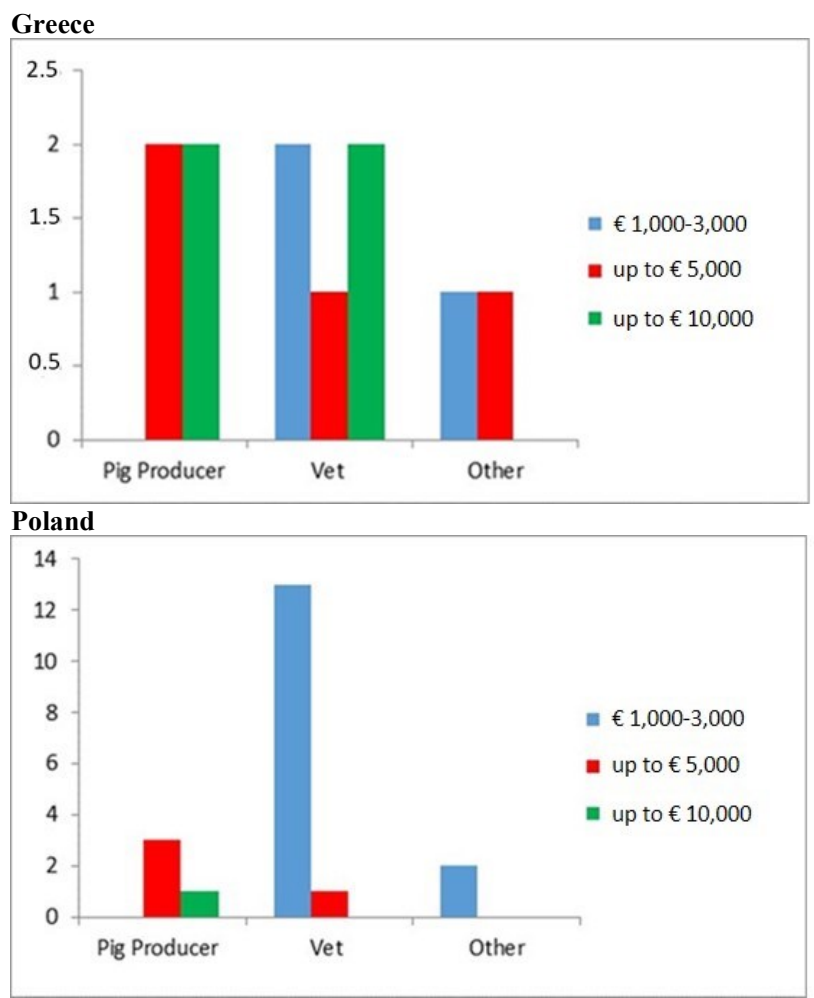

Fig. 4. Willingness of the different categories of stakeholder to invest money in diagnostic field devices, by cost bracket
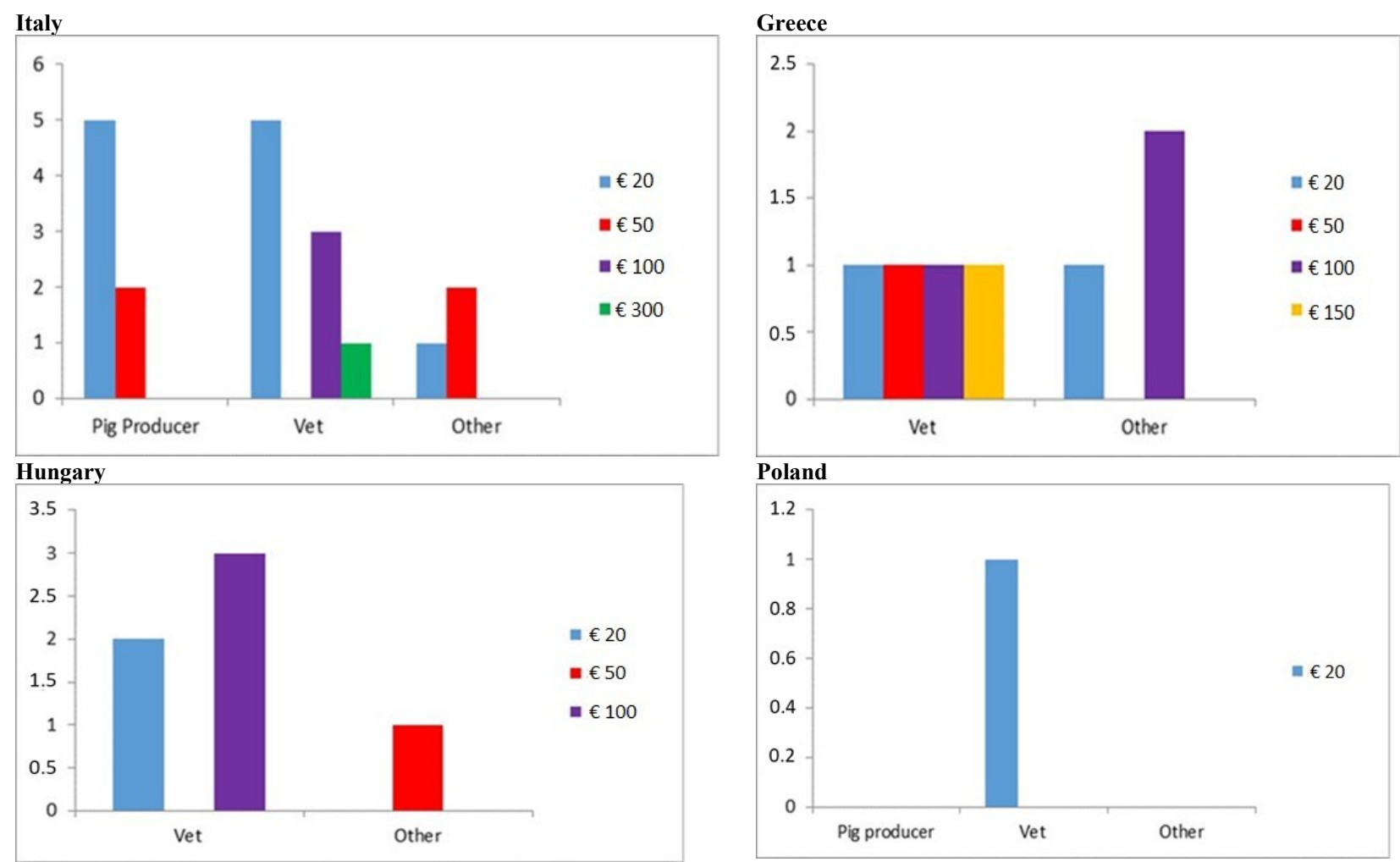

Fig. 5. Willingness of the different categories of stakeholders to rent the diagnostic field device, by rent amount 

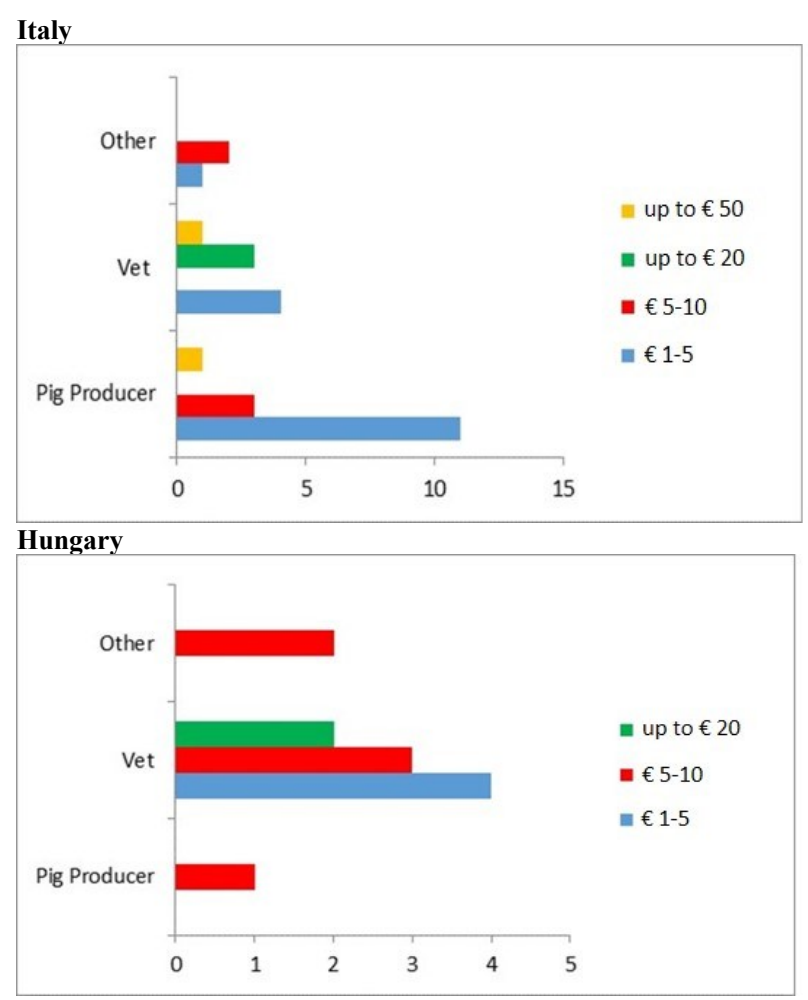
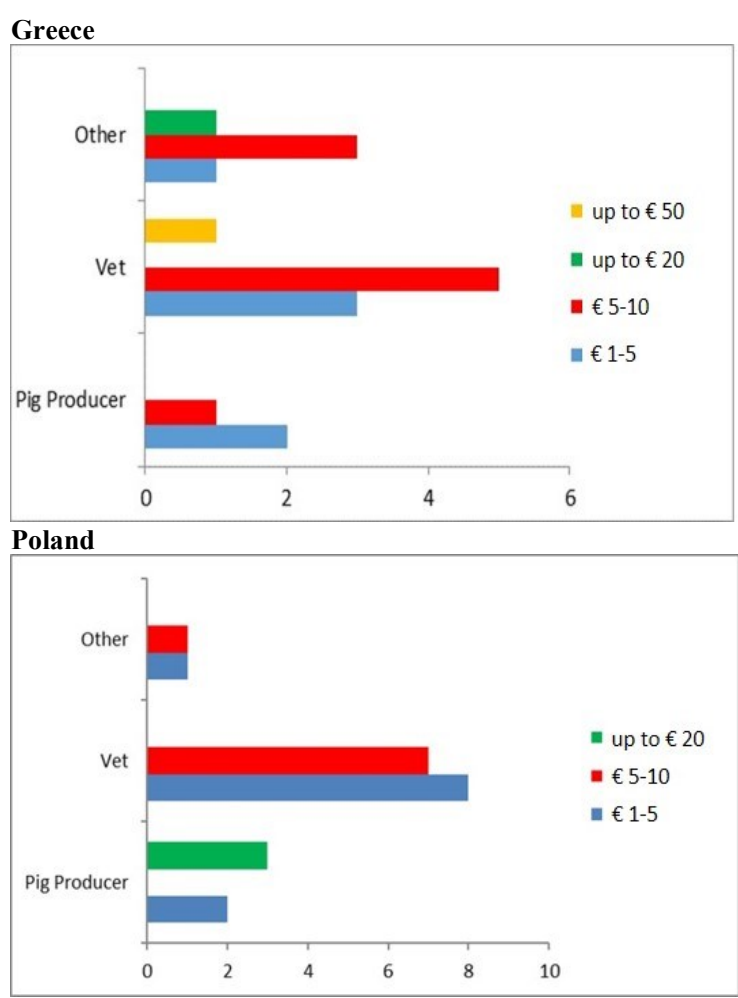

Fig. 6. Willingness of stakeholders to spend money for a single-use diagnostic kit, by unit cost

In Greece, the veterinarian group were evenly split on bearable monthly expenditure on rental, while in Hungary three professionals out of five were willing to spend $€ 100$. Regarding Poland, generally rental did not seem to be an interesting opportunity for stakeholders (Fig. 5).

When the tolerable cost for a single iteration of the diagnostic test for one of the six target diseases was investigated, in three out of four countries, veterinarians were the category which was most prepared to pay more, except in Poland, where pig farmers would spend higher amounts than them (Fig. 6).

\section{Discussion}

\section{Importance of the main diseases within the different countries}

Italy. The level of interest shown by stakeholders in PRRS diagnosis is because in Italy it is a well-known disease, capable of causing large losses on livestock farms due to abortions. This situation has been confirmed by studies conducted there which estimated the prevalence of infection by PRRS on Italian pig farms at around $90 \%$, but revealed infections to frequently manifest no clinical signs (3). The progressive acquisition of population immunity through natural infection or vaccination likely played a role in limiting transmission, but right now, it is not easy to estimate the real prevalence of the virus using serological tests because of the effects of vaccination.

PPV also seems to be a disease the diagnosis of which merits the attention of Italian stakeholders. This situation has been also demonstrated by a recent study consisting of a retrospective of PPV families isolated from archived Italian pig blood samples, which showed that several viruses of Parvoviridae family were present (2). The virus is prevalent nationally, so most intensive pig farms carry out double erysipelas and PPV vaccinations, which are very effective. Vaccination against PPV is also quite widespread on outdoor pig farms, but some of them still do not administer it to their animals.

The level of enthusiasm for PCV2 diagnosis directly depends on its connection to the occurrence of various clinical manifestations of PCV2 infection, typically the PCV2 systemic disease (PCV2-SD) formerly known as post weaning multisystemic wasting syndrome (PMWS), which used to be a widespread disease in Italy $(4,6,7)$. Other studies show that PCV2 is also distributed among the Italian wild boar population, which could represent a vector of infection, especially on open range pig farms (5).

Greece. In Greece, both veterinarians and pig producers had high anxiety over SIV, bearing out that the disease is currently circulating (with low pathogenicity strains) in many areas of the country, despite about $40 \%$ of sows being vaccinated annually. Recent epidemiological peer-reviewed studies on SIV 
do not exist, but field reports from private veterinarians indicate that approximately $25 \%$ of farms that do not perform vaccinations have seropositive sows for H1N1 and $10 \%$ have seropositive sows for H3N2 (personal communication).

Regarding PRRS, field reports show that $75 \%$ $80 \%$ of farms have infected animals. Many farms in the country vaccinate adult sows and boars but not growing piglets. Vaccination has been relatively successful in the last 4-5 years, but recently a small number of severe cases have been reported (personal communication).

Hungary. The results from Hungarian stakeholders for PRRS confirm the disease is present there. According to the latest data, the seroprevalence of PRRS is around $15 \%$ among the large herds, whereas the virus has been eliminated from backyard farms. Even though the eradication programme defines more and more areas as free from the PRRS virus, the pig-farming areas and counties most densely populated with animals are infected, and approximately $30 \%$ of the sows there live in infected herds (1).

In Hungary, PCV2 also seems to be an important disease to detect rapidly, and in fact, almost all farms vaccinate piglets (and/or sows) either with a viral subunit (in combination with Mycoplasma or alone) or with an inactivated whole-virus vaccine. The constant and long-term use of the vaccines, which are all based on genotype PCV2a, is causing a genotype shift. This has resulted in the appearance of PCV2b and recently PCV2d (Csagola, unpublished data). How this genetic change will affect the efficacy of the vaccines is still unclear, but the monitoring of the virus-induced disease and the need for reliable and quick diagnosis is evident.

SIV also seems to be extremely impactful upon stakeholders, and in fact, the disease seems to be present in almost every pig herd. There is no vaccination against SIV in Hungary, and the virus is frequently identified within cases of porcine respiratory disease complex.

Poland. In Poland, ASF was first confirmed in February 2014. The importance of this disease shown by the responses collected there is also confirmed by the overall disease situation, which is one of constant spread. To date, 3,000 cases of ASF in wild boars and 213 outbreaks of this disease in pigs have been detected (8).

PRRS is another important disease to be detected for Polish stakeholders. The estimated percentage of farms with seropositive animals reaches $80 \%$, and the virus strains currently circulating in pigs belong to subtype I and are considered mildly virulent (1). Despite the serious impact of the disease on the profitability of pig production, no more than $2 \%$ of sows are vaccinated. The frequent concurrent infections with Mycoplasma hyopneumoniae complicate the diagnosis and treatment.

The general summary from Polish responses is that four out of six targeted diseases are at the top of the end-users' priority list. The remaining diseases (CSF and ASF) are less well distributed, and this could explain the survey results. Nevertheless, ASF outbreaks are currently ongoing in some EU countries, having significant economic and health impacts.

\section{Diagnostic tool operational issues}

Touching on diagnostic tool operational issues, it is possible to observe that sensitivity is considered more important than a quick result and generally cheaper solutions are preferred, even if this would affect the portability of the device. Considering the heavy burden of responsibilities that pig farmers currently have to bear, simplicity is also considered an important parameter. These results may reflect their concerns about unmanageable new technology, which could potentially represent a further obstacle to efficient farming practice. They possibly consider fundamental that the instrument be easy to handle, in order to avoid a tedious learning process to master technical features and thus be able to devote time mainly to farming work.

\section{Economic issues}

Generally, veterinarians were seen to be willing to spend more than pig farmers both to buy or rent the instrument and on single tests. This could be linked to the possibility of having a field diagnostic tool capable of providing data in real time being an extremely important boon to a field veterinarian. This could allow them to drastically reduce the time needed to obtain results, allowing them to gain efficiency and productivity in their activities as professionals and possibly improve the quality and quantity of their work.

\section{Conclusions}

The survey results allowed the attitudes of the main potential end users of the device to be learned. These are expected to be private veterinarians and pig producers. Stakeholders from the four countries which participated in the survey in this preliminary phase seemed to be especially interested in the fast identification of PRRS, PCV2, SIV, and PPV, while ASF and CSF appeared to be less important because of their narrower diffusion in European countries at the time when the survey was conducted. Right now, especially for ASF diagnosis, the interest of stakeholders would probably be more important because of the continuous spread of the disease.

Functionality parameter data showed a pattern; the most important for those who answered were sensitivity, cost, simplicity, and time required to obtain results. It is important to note that sensitivity seemed 
more consequential than having a quick response. The assumption is that many of the end-users are not satsified with the currently available field diagnostic products, which might be not so reliable and sensitive, being able to detect a disease only at an advanced stage.

Concerning economic issues, the survey highlights very interesting information, useful in the drafting of a future business plan. In laying out such a plan, both selling and renting the device should be considered. The sum the market would be willing to spend is not an easy parameter to analyse for this technology product, due to the early development stage of the device at survey time (the participants did not have the chance to test the device). Nevertheless, it is possible to note that the majority of those who showed interest in buying the diagnostic tool with one single payment (about $53 \%$ of them) were willing to spend from $€ 1,000$ to $€ 3,000$, while nearly $33 \%$ were willing to spend up to $€ 5,000$ for a reliable point-of-care device.

Regarding the price of a single test, approximately $45 \%$ of the responders would accept paying between $€ 1$ and $€ 5$ while $38 \%$ would pay from $€ 5$ to $€ 10$ (for the panel of six analytes).

It is important to keep in mind that the questionnaires were collected at the beginning of the project, in order to form an initial idea of the perceptions of the stakeholders as to what would constitute attractive technical and economical parameters. The intention was to understand the issues of the end users and to compare their worries about epidemics to the actual diseases most widely diffused. This provided relevant input by which to set the future development path and produce a marketable analytical device. It also served to suggest possible changes to the target analytes to be included in potentially countryspecific panels. Additionally, the collected data and information are relevant input for the trade-offs which are unavoidable to make for the developers of any real-world analytical device. The technological implementations need to balance incurring the costs of the most cutting-edge, complex, and expensive solutions with serving the genuine needs and expectations of the market and suiting the device to the real conditions of use. Feedback from the system to the user must provide the most relevant information they expect.

The questionnaire collection and analysis are a completed research phase, but an additional questionnaire will be passed around the survey cohort at project end after the field tests, in order to collect the end-users' ultimate price anticipation and final evaluation of different aspects such as usability, analytical quality, etc.

Conflict of Interests Statement: The authors declare that there is no conflict of interests regarding the publication of this article.

Financial Disclosure Statement: The project "SWINOSTICS" is funded by Horizon 2020, the EU Framework Program for Research and Innovation for 2014-2020 under grant agreement no. 771649.

Animal Rights Statement: No animals were involved in the present study.

\section{References}

1. Balka G., Podgórska K., Brar M.S., Bálint Á., Cadar D., Celer V., Dénes L., Dirbakova Z., Jedryczko A., Márton L., Novosel D., Petrović T., Sirakov I., Szalay D., Toplak I., Leung F.C., Stadejek T.: Genetic diversity of PRRSV 1 in Central Eastern Europe in 1994-2014: origin and evolution of the virus in the region. Sci Rep 2018, 17; 7811. doi: 10.1038/s41598-018-26036-w.

2. Bovo S., Mazzoni G., Ribani A., Utzeri V.J., Bertolini F., Schiavo G., Fontanesi L.: A viral metagenomic approach on a non-metagenomic experiment: mining next generation sequencing datasets from pig DNA identified several porcine parvoviruses for a retrospective evaluation of viral infections. PLoS One 2017, 12, 1-17.

3. Franzo G., Dotto G., Cecchinato M., Pasotto D., Martini M., Drigo M.: Phylodynamic analysis of porcine reproductive and respiratory syndrome virus (PRRSV) in Italy: action of selective pressures and interactions between different clades. Infect Genet Evol 2015, 31, 149-157.

4. Marcato P.S., Sidoli L., Mandrioli L., Della Salda L., Cerati C., Rolla G.L.: Indagini clinico patologiche in un focolaio di PMWS (Postweaning multisystemic wasting syndrome) in suini del nord Italia. Large Anim Rev 1999, 5, 47-62.

5. Morandi F., Verin F., Sarli G., Canetti N., Scacco M., Panarese S., Poli A.: Immunolocalizzazione degli antigeni di circovirus suino tipo 2 (PCV2): diffusione dell'infezione e diagnosi di PMWS in popolazioni di cinghiale in Italia. Large Anim Rev 2010, 16, 77-84.

6. Petrini S., Paniccia M., Gavaudan S., Simoni E., Sensi M., Filipponi G., Rigotti L., Fortunati M., Ferrari M., De Mia G.M.: Porcine circovirus type 2 (PCV2)-associated diseases. Large Anim Rev 2011, 17, 89-98.

7. Sarli G., Ostanello F., Morandi F., Fusaro L., Bacci B., Nogrelli A., Alborali L., Dottori M., Vezzoli F., Barigazzi G., Fiorentini L., Sala V., Leotti G.: Diagnosis of PMWS in Italy. Large Anim Rev 2008, 14, 119-126.

8. Woźniakowski G., Kozak E., Kowalczyk A., Łyjak M., Pomorska-Mól M., Niemczuk K., Pejsak Z.: Current status of African swine fever virus in a population of wild boar in eastern Poland (2014-2015). Arch Virol 2016, 161, 189-195. doi: $10.1007 / \mathrm{s} 00705-015-2650-5$. Epub 2015 Oct 26. 